area specialization to ensure balance. About 50,000 titles will appear in the $3 \mathrm{~d}$ edition.

\section{Conclusion}

The year has also been an exciting one for me personally. I am grateful for the support of my ACRL and ALA friends as I move into a new position as director of libraries at Cleveland State University, and honored to have had the opportunity to serve as ACRL President.

\title{
CD-ROM: A primer
}

\author{
By Karen A. Becker \\ Circulation Librarian \\ Rosary College
}

\section{Some definitions, questions, and answers on}

a very hot topic.

$\mathbf{T}$ his primer evolved from a cheat sheet I prepared for myself to alleviate my confusion in dealing with the new optical/laser technology. After attending the Online ' 86 conference in Chicago, I realized how much I didn't know about CD-ROM and related technologies, and attempting to read the journal literature didn't help much. Authors assumed that the reader already understood the basics, such as: what is the difference between video disks and CD-ROM (there's a difference?), what are the "compatibility" problems to which everyone kept referring (compatible with what?), or what is really a "fair" price? The presentations and demonstrations were very amazing, but I wanted to be able to ask the salespeople and representatives some intelligent questions, and wished I was more familiar with the "burning issues."

This article is divided into two parts: the first part includes a "family tree" of optical technology and a list of definitions. As these applications of laser technology are fairly new, definitions do vary somewhat from source to source, but I have tried to synthesize the most commonly recognized definitions. The second part attempts to cover the issues currently being discussed with reference to optical technology, especially as it relates to libraries. The purpose of this paper is to instill confidence in the reader-confidence to read a journal article (even in a computer journal!) and confidence to communicate with publishers and vendors of optical technology.

\section{Optical disks}

Optical disk: Generic term for media produced and read with laser technology.

Laser disk: Optical disk.

Analog/digital: An analog signal has significance at all times. An analog (dial) watch can show any time, such as 8:32 and 37 seconds. Television transmissions use analog signals. A digital signal is measured as being either on or off, up or down, etc. A digital watch will show the time as being either $8: 32$ or $8: 33$. Digital signals are commonly used in computer programming languages. The important fact to remember from all this, is that some optical technology uses the analog format, and some the digital format.

How are optical disks made? The information is converted to the proper format (analog or digital) and written on a master disk with a laser (master- 


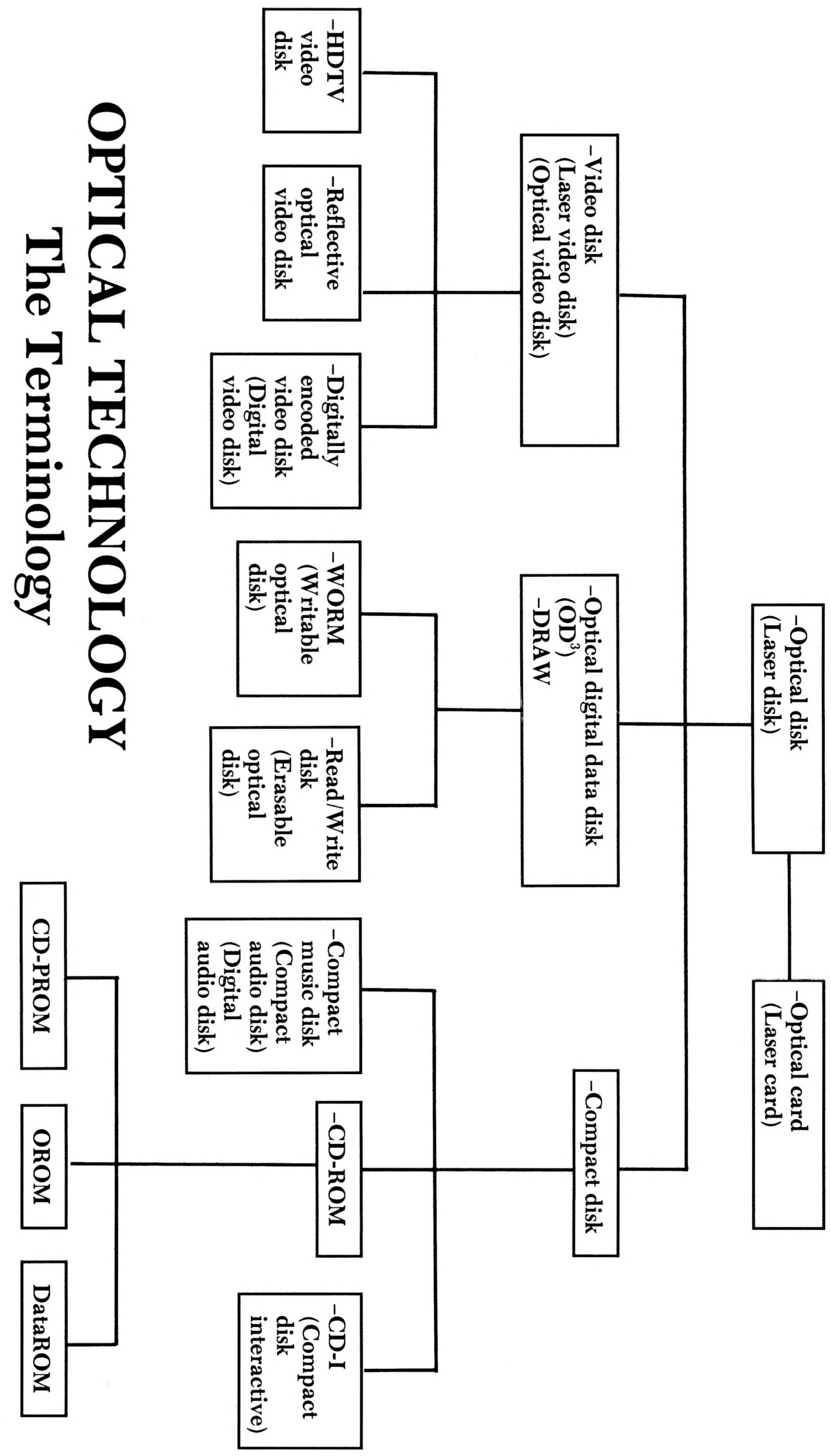


ing the disk). The master is made of glass, metal, or durable plastic, and the laser engraves or burns pits and bumps into the disk (in order to access the disk, another, lower power laser reads these pits and bumps). The most common method of replicating these disks is by stamping out copies from the master, similar to the process for making phonograph records. Each disk is protected from wear and tear by a clear plastic coating. The head of the optical disk reader (drive) does not touch the actual surface of the disk - a laser reads the disk through the coating. This gives the disks an estimated life of 10 years.

\section{Video disks}

Video disk: 12-inch disks usually recording sound and moving images such as motion pictures. The information is recorded on the disk in an ana$\log$ format - the standard format for television signals.

Laser video disk: Video disk.

Optical video disk: Video disk.

Reflective optical video disk: The video disk format most commonly used for recordings of movies. The disk has a silvery, rainbow color.

Digitally encoded video disk: Digital (machinereadable) data is converted to an analog format for recording on a video disk; this is the most economical format for recording still pictures, and is often used for interactive applications using motion pictures, still pictures, audio, and digital (textual) data.

Digital video disk: Digitally encoded video disk.

HDTV video disk: Video disks encoded with a signal readable by the new HDTV (high definition television). This experimental technology television has 1125 lines per screen rather than the conventional 230 to 525 lines.

\section{Optical digital data disks}

Optical digital data disk: A large, 12-inch disk recorded with digital information.

OD3: Optical digital data disk.

DRAW (Direct Read After Write): Each disk is written individually and sequentially (not stamped) and checked for errors as it is being written. If there is an error, the data is immediately rewritten. This process is time consuming, and can take $15 \%$ to $30 \%$ of the disk capacity.

WORM: Write Once, Read Many (or Mostly). User may write or record additional data, but cannot change data already input.

Writable optical disk: WORM.

Read/Write disks: Producers are experimenting with a disk that has the quality of erasability, permitting optical media to overcome the problems of read-only memory, and to compete more directly with magnetic media such as floppy disks or computer tape.

\section{Compact disks}

Compact disk: Data is recorded in digital form (machine readable) on disks $4^{3 / 4}$ inches in diameter. Compact disks can support audio (sound) and digital (textual) information. Video images are possible, but may not be economical since the analog signals must first be converted to digital form.

Compact audio disk: Stereo audio signals recorded in digital format. Each can record about 75 minutes of music.

Digital audio disk: Compact audio disk.

CD-ROM: Compact Disk-Read Only Memory. Can hold as much character-encoded digital data as 1,200 to 1,600 floppy disks, or 200 books of 300 pages each. Read Only Memory means that the data is permanently written on the disk - it cannot be added to, erased, rewritten, or altered.

CD-I: Compact Disk Interactive. A technology experimenting with disks storing video, audio, and digital (textual) information on one disk. These products are intended for the consumer mass market (educational, entertainment, etc.)

CD-PROM: Compact Disk-Programmable Read Only Memory. A CD with write once technology (see WORM).

DataROM: Sony's entry into the CD-ROM market is 5 inches, and will be erasable or write once data storage on one side, and permanent on the other.

OROM: 3M's entry into the optical technology market is 5 inches, and has write once capabilities.

\section{Laser card/Optical cards}

Laser card/Optical card: A related technology; the data is recorded on a small plastic card, and has write once capabilities.

\section{Questions}

What is the proper spelling, disk or disc? "Disc" is often used by producers and advertisers. However, the Wall Street Journal, the New York Times, the Encyclopaedia Britannica, and of course, Online have opted for "Disk."

What are the components of a CD-ROM system? 1) A computer such as an IBM PC; 2) Software to access the data; 3) An interface card (interface board); 4) Disk player (reader, drive); and 5) Compact disks.

Can all CD-ROM disks be read by all CD-ROM disk players? In theory, yes, but in real life, no. The disks and drives are manufactured to certain specifications of size and shape. However, CD-ROM producers have not established a standard for file formats and software, so the data can be organized

${ }^{1}$ Jeff Pemberton, "Shooting Ourselves in the Foot... and Other Consequences of Laserdisks," Online 10 (May 1986):9; and Jeff Pemberton, editor's note in Nancy Herther, "CDROM Technology: A New Era for Information Storage and Retrieval?" Online 9 (November 1985): 17. 


\section{Log on to}
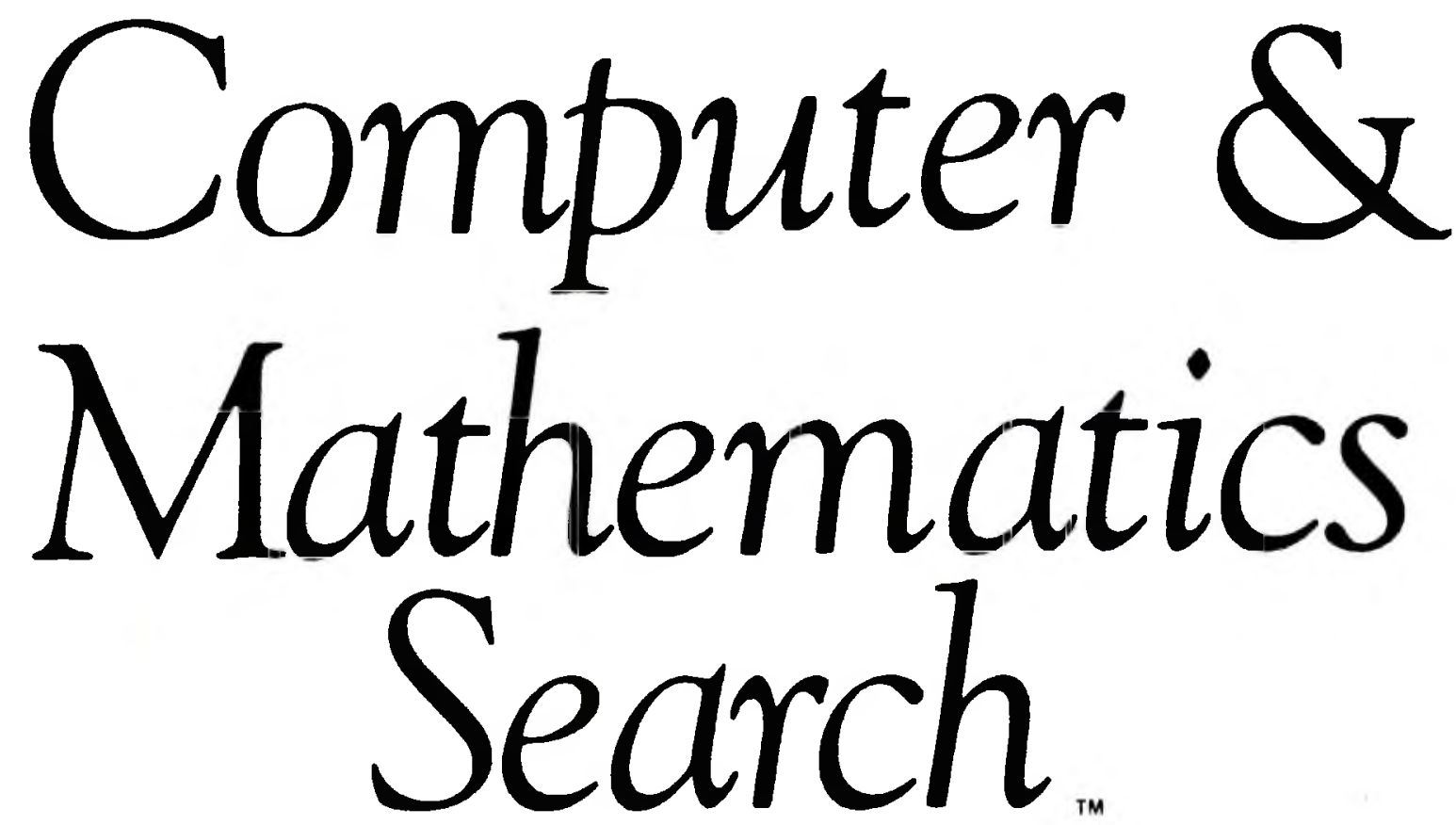

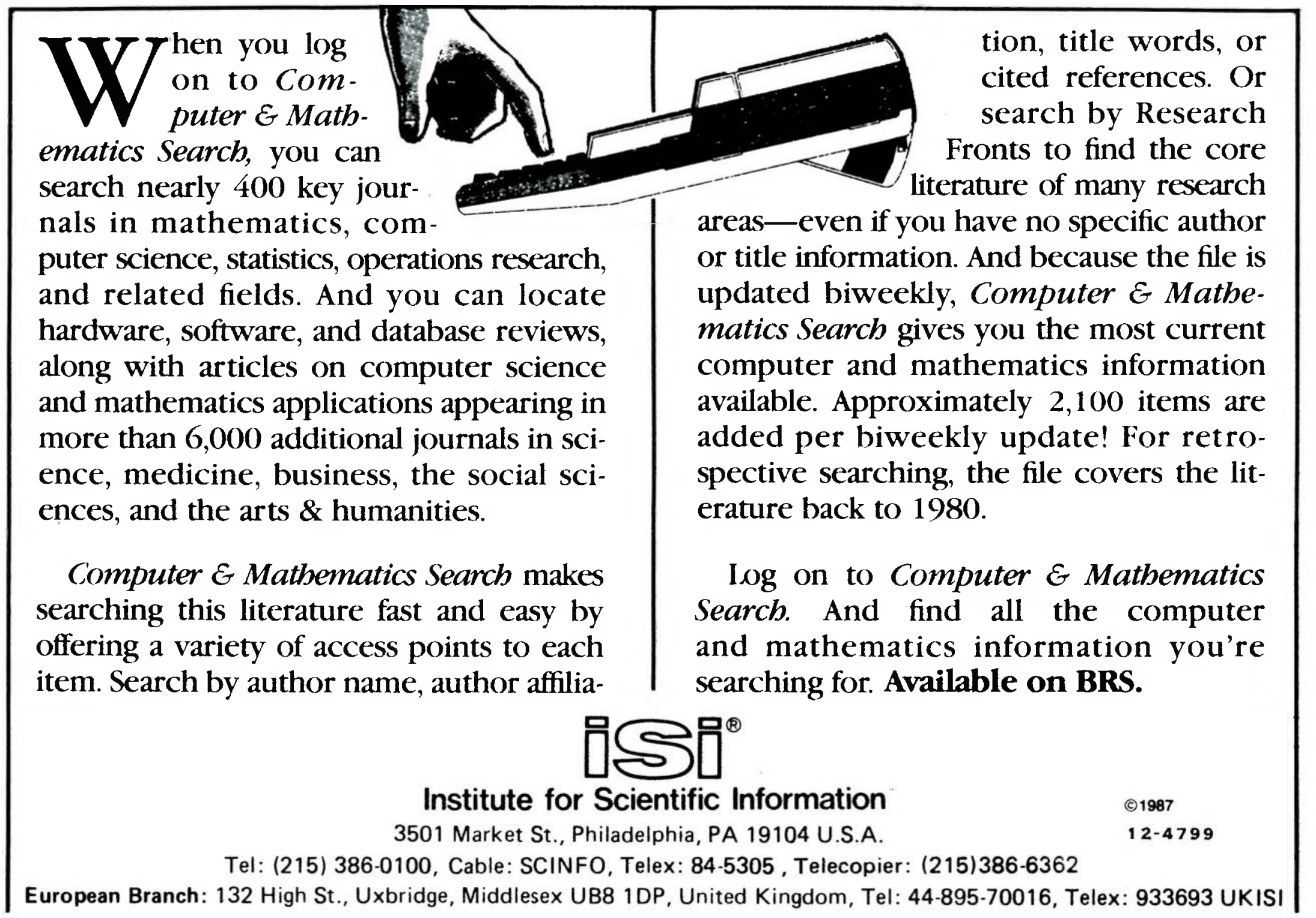


on the disks differently by each publisher. NISO (the National Information Standards Association) and the High Sierra Group (an ad hoc group of CDROM advocates) are working on setting these types of standards. There are also choices to be made regarding interface systems (see below). However, some people feel that it is too soon to set standards, that standards will stifle creativity and evolution of the technology. ${ }^{2}$

What does the interface card do? CD-ROM drives (players) need an interface system in order to link up with a microcomputer. Different CDROM publishers have developed different interfaces between selected computers (e.g., VAX, IBM $\mathrm{PC}$ ), and particular brands of CD players (e.g., Sony, Hitachi, or Philips). The three most common interfaces are SCSI (Small Computer Systems Interface), SASI (Shugart Associates Standard Interface), and Philips high-speed serial interface. The interface board is provided by the publisher. Nancy Herther notes, "The SCSI drive appears to have support as the de facto standard for interfacing the CD-ROM drives to microcomputers." 3

What are some related CD-ROM drive technologies? Large databases with multiple disks store the disks in a JUKEBOX, which works the same way as a phonograph record jukebox. The jukebox mechanically chooses and retrieves the disk containing the requested information and inserts it into an attached reader. A more recent innovation is "Philips' new 5-inch half-height CD-ROM drive which will fit into the floppy slot of a microcomputer."

Can I access databases on CD-ROM using the same commands I use online? Generally, yes. The CD-ROM publishers have adapted the access software from the online systems. However, the more advanced commands may not be available for use (for example, proximity searching with "N"). Some systems, such as InfoTrac, do not offer Boolean searching at all. The search and retrieval software is provided by the publisher. Also be aware that databases on CD-ROM don't always contain all the backfiles of the online database. Some, such as ERIC on Silverplatter, permit the user to purchase the disks containing the backfile, and replace only the current disk quarterly. Dissertation Abstracts on CD will contain from 1861 to the present, but Compact Medline has only about 2 years of data.

What kind of computer do I need? Each CDROM product has its own specification. Types of computers commonly listed include Apple, VAX, IBM PC, and IBM PC compatibles, and may require a minimum amount of memory such as $256 \mathrm{~K}$ or $512 \mathrm{~K}$. Some especially large databases may require a hard disk drive.

${ }^{2}$ Optical/Electronic Publishing Directory 1986 (Medford, N.J.: Learned Information, 1986), pp. $14,21-22$.

${ }^{3}$ Nancy Herther, "CDROM and Information Dissemination: An Update," Online 11 (March 1987):59.

${ }^{4}$ Ibid., p. 60 .
What type of information is available for libraries in the CD-ROM format? 1) Support tools such as BiblioFile (1.4 million MARC records), Books in Print, Ulrich's; 2) Databases such as ERIC and Medline; and 3) Reference tools such as the Academic American Encyclopedia and the Oxford English Dictionary (1987).

What is the price range for CD-ROM products? Expensive!! Grolier Electronic Encyclopedia has a one-time charge of $\$ 199$ for the disk, but the interface card is an additional $\$ 395$ and the search software another $\$ 199$, so be careful to check what is included and what is extra! Examples of annual subscription charges include Books in Print Plus at $\$ 895$ per year, Ulrich's at $\$ 395$ per year, Compact Disclosure at $\$ 3,200$ per year, Dialog on Disc ERIC at $\$ 3,450$ per year, Compact Medline at $\$ 6,350$ per year, and Datext Corporate Information Database at $\$ 19,600$ per year! Be aware that some publishers are offering discounts for purchases of multiple copies or cooperative group purchases by libraries. Publishers also offer special lease, purchase, and rent-to-own deals on the CD players, but check around since prices for hardware are falling.

Why is it so expensive? Publishers insist that the process of creating the master is very expensive. However, some librarians feel that once the master is created, the publishers could recover their money faster by distributing more disks at a lower cost. Publishers also feel that they have no control over the data once it is sent to a library; theoretically it could be copied and distributed, especially with advances in WORM technology. Also, they have made a big investment in time, money, and resources on a still experimental technology, so they have to recoup that investment as quickly as possible. Information providers also raise the issue of the cost of the medium (the extrinsic costs of creating and replicating the disks), against the issue of the value of the contents (the intrinsic value of the information). ${ }^{5}$

Will prices be going down in the near future? Prices for hardware are predicted to drop, as audio CDs take hold in the consumer market, since audio CD players are manufactured with the same technology as CD-ROM players. And, of course, prices for IBM PC compatibles are very reasonable. Prices for databases are another matter. Some information providers adamantly state that prices will not fall significantly, while others admit that they may be pricing themselves out of the market (ERIC on Silverplatter has recently dropped its

${ }^{5}$ Herther, "CDROM and Information Dissemination," p. 59; Pemberton, "Shooting Ourselves," p. 10; Nancy Herther, "A Light in Your Future: Market and Technology Trends in Optical Storage, an Interview with Edward S. Rothchild," Online 11 (January 1987):129; David C. Miller, "Laser Disks at the Library Door: The Microsoft First International Conference on CD-ROM," Library Hi Tech 4 (Summer 1986):58-59. 
prices from the introductory rate of $\$ 1,750$ to $\$ 650$ annually for the quarterly updates, and from $\$ 2,000$ to a $\$ 900$ one-time charge for their backfiles).

Could libraries share disks? Probably not, but again this may differ with each publisher. When asked about their policies concerning conditions of usage, publishers gave answers ranging from "no restrictions" to "copyrighted" to "public domain" to "standard software licensing agreement" to "use restricted to workstation level." Information providers expressing concern over how to maintain control over "their" data need to work on this issue with librarians.

\footnotetext{
${ }^{6}$ Helen Gordon, editor's note in Bruce Connolly, "Laserdisk Directory: Part I," Database 9 (June 1986): 15 .
}

Is this technology here to stay, or is this just a fad? Do you think the glass is half-empty or halffull? No one can predict what will happen with optical technology. New processes and applications are being discovered all the time. The new CD-I formats may influence the CD-ROM formats. Prices for CD-ROM databases may fall to reasonable levels. Omnidrives which can play CDs, video formats, and WORM formats may be invented. ${ }^{7}$ The situation in some libraries may indicate that using certain databases on CD-ROM is less expensive than accessing them online. What do you think?

${ }^{7}$ Optical/Electronic Publishing Directory 1986, p. 19.

\section{Planning for CD-ROM in}

\section{the Reference Department}

\author{
By Gail T. Graves \\ Head, Reference Department \\ University of Mississippi
}

and Beth F. King

Systems Librarian

University of Mississippi

\author{
Laura G. Harper \\ Head, Online Search Services \\ University of Mississippi
}

How to select software and equipment.

$\mathbf{T}$ he decision to purchase CD-ROM databases requires a commitment from librarians to adapt to a new technology, to approach bibliographic instruction from a new perspective, and to stay abreast of developments in a rapidly changing field. This decision cannot be made hastily. Implementing this new service requires an initial commitment of resources for equipment and a revision of the materials budget to absorb considerable on- going expenses.

The Williams Library at the University of Mississippi has a centralized Reference Department that provides primary service to all disciplines, with the exception of law, pharmacy, chemistry, and music. The library has offered a fee-based search service for ten years and acquired InfoTrac two years ago. The head of reference and the online search coordinator had observed the development 